Revista Calidad en la Educación Superior

Programa de Autoevaluación Académica

Universidad Estatal a Distancia

ISSN 1659-4703

Costa Rica

revistacalidad@uned.ac.cr

\title{
LA ASESORÍA DEL PROGRAMA DE APOYO CURRICULAR Y EVALUACIÓN DE LOS APRENDIZAJES (PACE) EN TORNO A LA EVALUACIÓN DE LOS APRENDIZAJES: ALCANCES Y RETOS DESDE LA PLANIFICACIÓN CURRICULAR INSTITUCIONAL.
}

THE ADVICE AROUND THE LEARNING EVALUATION OF THE PROGRAMA DE APOYO CURRICULAR Y EVALUACIÓN DE LOS APRENDIZAJES (PACE): ACHIEVEMENTS AND CHALLENGES FROM INSTITUTIONAL CURRICULUM PLANNING.

Ana Cristina Umaña Mata, acumanam@uned.ac.cr ${ }^{1}$ Xinia Calvo Cruz, xcalvo@uned.ac.cr ${ }^{2}$ Natalia Salas Quirós, nsalas@uned.ac.cr ${ }^{3}$ Laura Jiménez Aragón, Ijimeneza@uned.ac.cr ${ }^{4}$ Suzanne Soto Soto, ssoto@uned.ac.cr ${ }^{5}$ Gisselle Gómez Avalos, ggomez@uned.ac.cr ${ }^{6}$ Melba Fallas Zúñiga, mfallas@uned.ac.cr ${ }^{7}$ Carolina Tencio Blanco, ctencio@uned.ac.cr ${ }^{8}$ Programa de Apoyo Curricular y Evaluación de los Aprendizajes Universidad Estatal a Distancia, Costa Rica Aprender para evaluar y Evaluar para Aprender I Simposio sobre Evaluación de los Aprendizajes

Volumen 5, número 2

Noviembre, 2014

Recibido: agosto, 2014

pp. 214 - 238

Aprobado: octubre, 2014

\footnotetext{
${ }^{1}$ Doctora en Educación, Máster en Planificación Curricular, Coordinadora del Programa de Apoyo Curricular y Evaluación de los Aprendizajes

2 Máster en Planificación Curricular, Licenciada en Educación Primaria, Asesora Curricular del PACE.

${ }^{3}$ Máster en Planificación Curricular, Licenciada en Administración Educativa, Bachiller en Educación Preescolar, Asesora Curricular del PACE.

${ }^{4}$ Máster en Currículo y Docencia Universitaria, Asesora Curricular del PACE.

${ }^{5}$ Máster en Planificación Curricular, Asesora Curricular del PACE.

${ }^{6}$ Licenciada en Enseñanza de los Estudios Sociales y Educación Cívica Máster en Planificación Curricular, Asesora Curricular del PACE.

${ }^{7}$ Máster en Planificación Curricular, Licenciada en Educación Primaria, Asesora Curricular del PACE. 8 Máster en Planificación Curricular, Licenciada en Ciencias de la Educación con énfasis en I y II Ciclos, Egresada de la Licenciatura en Administración Educativa.
} 
La asesoría del Programa de Apoyo Curricular y Evaluación de los Aprendizajes (PACE) en torno a la evaluación de los aprendizajes: alcances y retos desde la planificación curricular institucional

Ana Cristina Umaña, Xinia Calvo, Natalia Salas, Laura Jiménez, Suzanne Soto, Gisselle Gómez, Carolina Tencio y Melba Fallas

\title{
Resumen
}

El Programa de Apoyo Curricular y Evaluación de los Aprendizajes (PACE) de la Universidad Estatal a Distancia (UNED) es la instancia que asesora a nivel institucional en el ámbito curricular. Como parte de la asesoría, este Programa considera la evaluación de los aprendizajes, tema prioritario en la educación a distancia y sobre el cual se centrará la presente sistematización.

A la fecha, el personal de esta instancia hace esfuerzos sustantivos mediante la investigación de la temática, en aras de generar conocimientos para asesorar procesos y emitir criterios curriculares a las diversas solicitudes realizadas, así como elaborar documentos que apoyen la labor pedagógica en el ámbito mencionado, entre otros aspectos de importancia.

Palabras claves: asesoría curricular, evaluación de los aprendizajes, diseño curricular, criterio curricular, producción académica.

\begin{abstract}
Programa de Apoyo Curricular y Evaluación de los Aprendizajes (PACE) of the Universidad Estatal a Distancia (UNED), is the department in charge of the curriculum advisory at institutional level. As part of the advice that performs, it is considered the evaluation of learning a priority in distance education and the intention of this systematization will focus on that.

To date this instance staff makes substantive efforts through research on the topic, in order to generate knowledge to advice processes, give curricular criteria to different requests, as well as the elaboration of documents that support the educational work in the area in mention, among other things of importance.
\end{abstract}

Keywords: curriculum advisory, evaluation of learning, curriculum design, curricular criteria, academic production.

\section{Introducción}

EI PACE de la Universidad Estatal a Distancia es la instancia que asesora a nivel institucional en el ámbito curricular. En este sentido, corresponde desde su quehacer asumir diversos ámbitos; siendo comunes la asesoría de diseño de planes de estudios, asignaturas y cursos, la elaboración de documentos que orienten la labor docente y la realización de actividades solicitadas por equipos 
La asesoría del Programa de Apoyo Curricular y Evaluación de los Aprendizajes (PACE) en torno a la evaluación de los aprendizajes: alcances y retos desde la planificación curricular institucional

Ana Cristina Umaña, Xinia Calvo, Natalia Salas, Laura Jiménez, Suzanne Soto, Gisselle Gómez, Carolina Tencio y Melba Fallas

preocupados por alguna temática curricular específica de un plan de estudios, de una asignatura, de un programa, de una cátedra o inclusive de estudiantes en particular, entre otras muchas especificidades en torno al tema curricular.

Como parte de las asesorías que brinda dicha instancia destaca la evaluación de los aprendizajes, tema prioritario en la educación a distancia y objeto de interés de la presente sistematización. Sobre este particular, se indica que además de la asesoría en la propuesta evaluativa de los planes de estudios y asignaturas es frecuente ser partícipes de procesos para resolver apelaciones del estudiantado que surgen a partir de las prácticas evaluativas planificadas. En este sentido es importante acotar que dichas apelaciones se canalizan generalmente a través de la Defensoría de los Estudiantes, sin embargo se recurre a la instancia en busca de un insumo técnico de importancia para la toma de decisiones de cada caso en particular. De este modo, se ha logrado articular una colaboración por parte del PACE, brindando asesoría oportuna, relevante y actualizada en lo que respecta a este tipo de demandas y apoyando, en beneficio de las prácticas evaluativas, la calidad académica y del proceso educativo en el tema de evaluación de los aprendizajes.

Ahora bien, como parte del diario acontecer en torno a la evaluación de los aprendizajes, el PACE —en atención a las demandas del contexto y en afán de un mejoramiento continuo - ha sentido la necesidad de elaborar documentos para apoyar la labor de asesoría en la temática específica de evaluación de los aprendizajes. De esta forma, desde su creación en el año 2007, la instancia ha sido gestora y partícipe de procesos de construcción de documentos que han fortalecido y orientado el quehacer académico en la UNED. Estos procesos de construcción han permitido no solo tener un lenguaje común, sino además el establecimiento de líneas de acción en un sistema en el que intervienen diversos 
La asesoría del Programa de Apoyo Curricular y Evaluación de los Aprendizajes (PACE) en torno a la evaluación de los aprendizajes: alcances y retos desde la planificación curricular institucional

Ana Cristina Umaña, Xinia Calvo, Natalia Salas, Laura Jiménez, Suzanne Soto, Gisselle Gómez, Carolina Tencio y Melba Fallas

agentes y momentos en el proceso de mediación docente, que demandan, por tanto, claridad de los principios teórico-prácticos de la evaluación de los aprendizajes que se lleva a cabo en un sistema de educación a distancia.

A la fecha, el personal del PACE realiza esfuerzos sustantivos mediante la investigación (formación y actualización) en la temática de evaluación de los aprendizajes, en aras de generar conocimientos y hacerle frente a los retos y las demandas de la asesoría requerida en la educación a distancia, específicamente en este campo.

El presente documento tiene como objetivo sistematizar la experiencia de la asesoría curricular del PACE en torno a la evaluación de los aprendizajes, en un afán de plasmar los alcances y retos que se tienen desde la planificación curricular institucional.

\section{Antecedentes}

El Programa de Apoyo Curricular y Evaluación de los Aprendizajes (PACE) de la Universidad Estatal a Distancia fue creado en el año 2006. Desde su campo de acción se ocupa de variados espacios en pro del desarrollo y de la mejora académica.

EI PACE, en su Plan Estratégico del Programa de Apoyo Curricular y Evaluación de los Aprendizajes 2007-2012 (UNED, 2007, pp.4-5), propone como objetivos los siguientes:

- Desarrollar acciones de asesoramiento y capacitación en materia curricular y evaluación de los aprendizajes para acompañar a los 
La asesoría del Programa de Apoyo Curricular y Evaluación de los Aprendizajes (PACE) en torno a la evaluación de los aprendizajes: alcances y retos desde la planificación curricular institucional

Ana Cristina Umaña, Xinia Calvo, Natalia Salas, Laura Jiménez, Suzanne Soto, Gisselle Gómez, Carolina Tencio y Melba Fallas

programas de extensión, pregrado, grado y posgrado, por medio de estudios, planes experimentales, investigaciones y actividades de mejoramiento

- Coadyuvar en la producción, la continuidad y el máximo aprovechamiento de los conocimientos en torno a los problemas y el mejoramiento de los procesos de enseñanza-aprendizaje que favorezcan la toma de decisiones en el currículo universitario.

- $\quad$ Articular las diferentes funciones del programa con aquellas unidades académicas vinculadas al diseño, desarrollo y evaluación del currículo y de los aprendizajes, para llevar la oferta académica universitaria hacia la mejora continua y la búsqueda de la excelencia

- $\quad$ Articular el proceso de planificación, ejecución y evaluación curricular en los programas extensión, pregrado, grado y posgrado de la universidad - Generar espacios académicos que favorezcan el intercambio de experiencias y conocimientos para el crecimiento profesional de los miembros de la Comunidad Universitaria.

Con la intención de homologar criterios, es importante tener claro lo que se entiende por asesoría. Al respecto, Lucarelli (2008) indicó que:

La incorporación de la asesoría pedagógica universitaria es vista como una estrategia que permite la orientación de los procesos académicos hacia el éxito de las metas y objetivos requeridos para la transformación de la universidad como un espacio para la formación, investigación y acción social para los nuevos tiempos (citado por Calvo, Fallas, Gómez, Jiménez, Salas, Robles, Soto, Tencio y Umaña, 2011, p. 5).

Aunado a lo anterior, Calvo et al. (2011) indicaron que autores como Brovelli (2000) y Orozco (2007) se refieren a esta asesoría como asesoría curricular; la cual es desempeñada por un profesional "que ofrece formación y asesoramiento 
La asesoría del Programa de Apoyo Curricular y Evaluación de los Aprendizajes (PACE) en torno a la evaluación de los aprendizajes: alcances y retos desde la planificación curricular institucional

Ana Cristina Umaña, Xinia Calvo, Natalia Salas, Laura Jiménez, Suzanne Soto, Gisselle Gómez, Carolina Tencio y

Melba Fallas

en problemas específicos de este campo de conocimiento" (Brovelli, 2000, p. 75 citado en Calvo et al., 2011).

En relación con el tema, se destaca que en la UNED el PACE es la instancia que se ocupa de la asesoría curricular a nivel institucional. En este punto es importante destacar que los primeros tres años del Programa estuvieron abocados a la asesoría de los diseños y rediseños de los planes de estudios y asignaturas; sin embargo, los últimos tres años han obligado a su personal a prepararse intensamente en variados temas curriculares, ya que las demandas de asesoría son cada vez más constantes y variadas. En consecuencia, el personal se encuentra inmerso en una dinámica continua de formación y autoformación en temáticas curriculares como son la internacionalización, la flexibilidad, los paradigmas, los enfoques, las metodologías, el aprendizaje mediante plataforma virtual y la evaluación de los aprendizajes, entre otras tantas que impulsan el quehacer de la instancia como un espacio propicio de apoyo para el mejoramiento de las instancias académicas institucionales.

Sobre el tema específico de evaluación de los aprendizajes, razón de ser de la presente sistematización, es importante destacar que el PACE se encuentra en un proceso arduo de trabajo en torno al tema, lo que ha demandado la formación a lo interno del equipo y la incorporación de procesos de investigación que permitan trascender las prácticas evaluativas tradicionales y, en su lugar, se abran espacios para la evaluación alternativa, como una opción que se enmarque en aprender y en la naturaleza misma de la educación a distancia.

En adelante, el presente escrito se ocupa de exponer por apartados la experiencia del PACE en torno a la asesoría de evaluación de los aprendizajes desde un plan de estudios o un diseño o rediseño de asignatura y el pronunciamiento curricular 
La asesoría del Programa de Apoyo Curricular y Evaluación de los Aprendizajes (PACE) en torno a la evaluación de los aprendizajes: alcances y retos desde la planificación curricular institucional

Ana Cristina Umaña, Xinia Calvo, Natalia Salas, Laura Jiménez, Suzanne Soto, Gisselle Gómez, Carolina Tencio y Melba Fallas

del PACE en los procesos de resolución de las apelaciones relacionadas con los procesos de evaluación de aprendizajes, así como la participación y producción en procesos de mejora en el área de interés de la presente sistematización. Finalmente, se le dedica un espacio al planteamiento de las líneas institucionales que orientan la acción del PACE en materia de evaluación de los aprendizajes.

\section{Desarrollo de la sistematización de la experiencia del PACE}

\section{La asesoría curricular de la propuesta evaluativa de un diseño o rediseño del plan de estudio o de la asignatura de la UNED.}

El mundo de hoy le exige a la educación superior responder a sus demandas e intereses desde procesos formativos pertinentes, viables y de calidad. En este sentido, dicho nivel educativo está en la obligación de responder con propuestas que apunten hacia logros de impacto social. Al respecto, Camelo (2012) destacó que el mundo y la sociedad que lo habita están en continuo cambio y que los sistemas educativos se han reformado para adaptarse a las necesidades actuales, demandas económicas, tecnológicas, científicas y también sociales. En cuanto a este tema, la UNED realiza esfuerzos sustantivos desde sus propuestas de planes de estudios para cumplir con las demandas indicadas en aspectos tales como el perfil profesional, la metodología de su oferta y la propuesta evaluativa del aprendizaje, así como todos y cada uno de los aspectos curriculares que son parte de los lineamientos institucionales que enmarcan la labor de la persona asesora curricular.

De esta manera, a la persona asesora curricular le corresponde generar un vínculo entre el campo de conocimiento específico que asesore y la teoría y 
La asesoría del Programa de Apoyo Curricular y Evaluación de los Aprendizajes (PACE) en torno a la evaluación de los aprendizajes: alcances y retos desde la planificación curricular institucional

Ana Cristina Umaña, Xinia Calvo, Natalia Salas, Laura Jiménez, Suzanne Soto, Gisselle Gómez, Carolina Tencio y Melba Fallas

técnica curricular de su dominio, con el fin de ofrecer pertinencia desde la oferta curricular.

Ahora bien, desde cada institución se tienen lineamientos curriculares que enmarcan la labor de la persona asesora. En el caso de la UNED, la asesoría del PACE se orienta hacia la necesidad de que los equipos de trabajo — conformados según lo que se establece en el artículo 13 del Reglamento de Gestión Académica (UNED, 2005) - planteen un proceso de evaluación de los aprendizajes que responda, en primer término, al planteamiento asumido por el Modelo Pedagógico institucional (UNED, 2004), es decir la "regulación y autorregulación de los aprendizajes, de manera que llegue a ser integral durante todo el proceso de aprender, e integrada, es decir no separada del proceso como momento de comprobación" (p. 36).

En consecuencia con lo anterior, no solo se asesora para el logro de una propuesta de evaluación sumativa, cuyo fin último es la acreditación de los aprendizajes mediante valores asignados, sino además, y con mayor importancia, la evaluación formativa, entendida como acción reguladora y autorreguladora de aprendizajes, así como la evaluación diagnóstica, la que con regularidad es comprendida como inicial en los procesos de aprendizaje, cuando su papel real es el de obtener información para orientar, direccionar o redireccionar procesos de aprendizaje en función de objetivos por lograr.

Finalmente, la asesoría curricular lleva al equipo de responsables a concretar la forma en que la mediación pedagógica del plan de estudios concretará la autoevaluación, la heteroevaluación y la evaluación entre pares. 
La asesoría del Programa de Apoyo Curricular y Evaluación de los Aprendizajes (PACE) en torno a la evaluación de los aprendizajes: alcances y retos desde la planificación curricular institucional

Ana Cristina Umaña, Xinia Calvo, Natalia Salas, Laura Jiménez, Suzanne Soto, Gisselle Gómez, Carolina Tencio y Melba Fallas

A manera de síntesis, la asesoría curricular del plan de estudios en materia de evaluación de los aprendizajes se concreta en las respuestas a las siguientes interrogantes: ¿Cuáles serán los momentos en los que se realizará la evaluación de los aprendizajes? ¿Cómo se concibe la evaluación diagnóstica, la formativa y la sumativa? ¿Cómo se concibe la autorregulación y la regulación de los aprendizajes y quiénes son las personas responsables? ¿Cuáles estrategias, técnicas e instrumentos se utilizarán como parte del proceso de evaluación de los aprendizajes?

Siguiendo esta línea, y sin dejar de lado lo establecido en los planes de estudios, la asesoría en materia de evaluación de los aprendizajes continúa en el nivel meso de planificación curricular, que corresponde al diseño de las asignaturas del plan de estudios.

El diseño de asignaturas (...) es el nivel más operativo del plan de estudios en el cual se describen los objetivos y las estrategias de aprendizaje, los materiales didácticos por utilizar, así como el tipo de evaluación que aplicará. Contiene dos partes: la primera que refiere a las relaciones y características del curso; y la segunda correspondiente al detalle de los elementos curriculares (Corrales et al., 2006, citado por Programa de Apoyo Curricular y Evaluación de los Aprendizajes, 2013, p. 31).

En esta etapa la asesoría se dirige a la comisión encargada del diseño curricular, orientándola para que en el planteamiento de los elementos curriculares (objetivos, contenidos, experiencias de aprendizaje, materiales, recursos y evaluación) se tome en cuenta el enfoque curricular, la metodología y el enfoque evaluativo que, de manera general, se esbozaron en el plan de estudios.

Ahora bien, en los diseños de asignaturas hay dos espacios específicos en los que se da la asesoría curricular del PACE, a saber, uno en la primera parte del diseño curricular, a la que se le conoce como la plantilla de generalidades; aquí la 
La asesoría del Programa de Apoyo Curricular y Evaluación de los Aprendizajes (PACE) en torno a la evaluación de los aprendizajes: alcances y retos desde la planificación curricular institucional

Ana Cristina Umaña, Xinia Calvo, Natalia Salas, Laura Jiménez, Suzanne Soto, Gisselle Gómez, Carolina Tencio y Melba Fallas

asesoría curricular se orienta hacia la descripción del apartado denominado "Propuesta de la evaluación de los aprendizajes".

En esta parte se consideran las evaluaciones de los aprendizajes diagnóstica, formativa y sumativa. En el caso de las dos primeras la asesoría se dirige hacia la reflexión y la toma de decisiones acerca de los momentos, las acciones y las estrategias mediante las cuáles se aplicarán estas dos funciones de la evaluación durante la implementación de la asignatura o del curso. En el caso de la evaluación sumativa se orienta a las personas responsables, para que planteen una propuesta de las actividades de evaluación (técnicas, estrategias e instrumentos de evaluación) que se utilizarán. Se indican en el diseño los rubros de calificación para cada una de las actividades propuestas.

El otro comprende la segunda parte del diseño curricular, que es el planteamiento medular de los elementos curriculares. En esta plantilla se planea cada uno de los objetivos de aprendizaje, con los respectivos contenidos, las experiencias de aprendizaje, los materiales, los recursos y la evaluación. En esta etapa se asesora para que se indiquen en la columna correspondiente a la evaluación los aprendizajes que se espera que el estudiantado haya alcanzado, como resultado del proceso de aprendizaje evidenciado en la columna de experiencias de aprendizaje y de acuerdo con el nivel taxonómico de conocimiento expresado en el verbo indicado en el objetivo.

La asesoría dirige a las personas responsables para que el planteamiento del proceso de evaluación sea coherente, no solo con el plan de estudios, como ya se indicó, sino que también sea coherente e integrado al resto de elementos curriculares planteados en la asignatura. De tal manera que la evaluación no se 
La asesoría del Programa de Apoyo Curricular y Evaluación de los Aprendizajes (PACE) en torno a la evaluación de los aprendizajes: alcances y retos desde la planificación curricular institucional

Ana Cristina Umaña, Xinia Calvo, Natalia Salas, Laura Jiménez, Suzanne Soto, Gisselle Gómez, Carolina Tencio y Melba Fallas

vea como un proceso aparte de los procesos de enseñanza y aprendizaje, sino más bien como un proceso integrado a estos.

Finalmente, es sustancial señalar que, como parte de esta experiencia de asesoría de diseño de planes de estudios y asignaturas, la temática de evaluación de los aprendizajes le ha demandado a los equipos responsables generar espacios de reflexión que fundamenten sus propuestas curriculares y permitan tomar decisiones concienzudas respecto a la administración del currículo desde los diferentes niveles y actores involucrados. Aunado a lo anterior, vale destacar que en diversas ocasiones las personas responsables han requerido de asesoría específica para la implementación de alguna de las estrategias de evaluación de los aprendizajes considerados en la planificación curricular, lo que sin lugar a dudas favorece, ya que responde a necesidades sentidas a nivel particular y por tanto a públicos meta específicos.

\section{El criterio curricular del PACE en el proceso de resolución de las apelaciones sobre evaluación de aprendizajes.}

Además de los procesos de asesoría, entre las funciones del PACE está velar por que las propuestas educativas guarden la coherencia curricular necesaria, entendida como "la relación lógica que deben guardar cada uno de los componentes curriculares entre sí, de manera que responda al enfoque curricular seleccionado para el proceso educativo" (PACE, 2013, p. 27). Dicha coherencia atiende de igual manera a la evaluación de los aprendizajes, como elemento curricular que tiene importancia en el sistema de educación a distancia y que constituye el tema central del presente documento. 
La asesoría del Programa de Apoyo Curricular y Evaluación de los Aprendizajes (PACE) en torno a la evaluación de los aprendizajes: alcances y retos desde la planificación curricular institucional

Ana Cristina Umaña, Xinia Calvo, Natalia Salas, Laura Jiménez, Suzanne Soto, Gisselle Gómez, Carolina Tencio y Melba Fallas

Esto significa que al emitir criterio sobre las apelaciones se debe tomar en cuenta la normativa institucional para la gestión académica y lo establecido por las teorías educativas respecto a la evaluación de los aprendizajes que se ajustan al Modelo Pedagógico, así como a la realidad de la educación a distancia en la UNED.

El debido proceso para efectuar las apelaciones correspondientes en la UNED se establece desde la ley n 6227, Ley General de la Administración Pública, en la que se indica que todo ente público debe tener lo que se llama doble instancia, que permite asegurar la existencia de dos recursos de defensa: revocatoria y apelación en subsidio. Estos dos recursos se encuentran contemplados en el Reglamento General Estudiantil (UNED, 2012) de la universidad en los artículos 61 y 62 , que indican el procedimiento a seguir para presentar la apelación.

Esta normativa institucional le permite al estudiantado optar por estas alternativas en caso de que algún resultado o proceso de evaluación de los aprendizajes de la asignatura que matriculó no sea satisfactorio y sea necesario considerar, en algún caso en particular, la situación con mayor detenimiento.

La Defensoría de los Estudiantes (DEFE) es entonces la instancia encargada de velar por que las apelaciones que surgen se resuelvan siguiendo lo que se establece en la normativa institucional. En este sentido, una vez resuelto un caso la única forma de retomar una apelación es aportar al caso pruebas y hechos nuevos que no fueron considerados anteriormente.

Como parte del marco de acción en el que la DEFE puede actuar, se ha encontrado pertinencia en realizar consultas al PACE como instancia asesora, para que desde su criterio experto en tema curricular y evaluación de los aprendizajes pueda aportar elementos relevantes en aquellas situaciones en las 
La asesoría del Programa de Apoyo Curricular y Evaluación de los Aprendizajes (PACE) en torno a la evaluación de los aprendizajes: alcances y retos desde la planificación curricular institucional

Ana Cristina Umaña, Xinia Calvo, Natalia Salas, Laura Jiménez, Suzanne Soto, Gisselle Gómez, Carolina Tencio y

Melba Fallas

que se considere necesario, permitiendo de esta manera aportar insumos para las decisiones que se tomen en los casos de apelación.

En este sentido, a la fecha las apelaciones presentadas por la DEFE al PACE se refieren únicamente a pruebas escritas aplicadas para el nivel de grado. Al respecto, se ha emitido criterio en cuanto a la validez técnica de la prueba en relación con el diseño, la extensión, la congruencia entre el nivel cognitivo indicado en los objetivos de aprendizaje, el nivel de dificultad del ítem y el tiempo designado para su ejecución. Otra forma en la que se atienden solicitudes de criterio curricular es por medio de la atención directa del estudiantado, que requiere una orientación para verificar si la apelación presentada es válida. Ocasionalmente, también se han atendido solicitudes del profesorado para poder resolver apelaciones que se les han presentado. Es importante resaltar que una apelación no necesariamente es presentada por un estudiante, también se atienden solicitudes de varios alumnos sobre un mismo caso.

Con la intención de formalizar las solicitudes se elaboró un documento que ha permitido recopilar la información necesaria para emitir los criterios en relación con las apelaciones presentadas. Desde la coordinación del PACE se reciben dichas solicitudes y se asigna a una de las asesoras del equipo de la instancia para que atienda y emita el dictamen curricular para ser entregado a la persona interesada.

De esta manera, en los casos presentados las asesoras curriculares han tomado en cuenta: el diseño curricular de la asignatura, las orientaciones académicas entregadas al estudiantado, la prueba escrita o el instrumento de evaluación referido y los materiales didácticos utilizados, así como la mediación pedagógica realizada a través de la plataforma virtual (en aquellas situaciones que la 
La asesoría del Programa de Apoyo Curricular y Evaluación de los Aprendizajes (PACE) en torno a la evaluación de los aprendizajes: alcances y retos desde la planificación curricular institucional

Ana Cristina Umaña, Xinia Calvo, Natalia Salas, Laura Jiménez, Suzanne Soto, Gisselle Gómez, Carolina Tencio y Melba Fallas

asignatura contemplara este medio para su oferta), entre otros aspectos, con el fin de verificar la validez del proceso de evaluación.

Dentro de los principales hallazgos de estos procesos de emisión de criterio curricular se encuentran los siguientes:

- Debido a la desactualización del diseño curricular de asignatura las orientaciones académicas no presentaban coherencia con este ni con lo que se pretendía evaluar.

- El nivel académico para el que fue diseñada la asignatura no correspondía con el nivel en el que fue ofertada. Es decir, una asignatura de licenciatura fue ubicada a nivel de bachillerato, lo cual significaba una limitante para el estudiantado en cuanto a los conocimientos previos que requerían.

- Hubo falta de claridad en los criterios de evaluación para que cada estudiante comprendiera qué se le estaba pidiendo.

- Existió falta de validez técnica de las pruebas escritas, pues no contaban con la tabla de especificaciones respectiva, además la redacción de las pruebas escritas, en algunos casos, no era clara, lo que tendía a confundir al estudiantado en sus respuestas. Asimismo, las instrucciones para realizar las actividades no precisaban lo que se esperaba que el estudiantado ejecutara.

- La realimentación que se le brindó a cada estudiante sobre su calificación no fue lo suficientemente clara y precisa para orientarlo respecto al resultado de aprendizaje esperado de su parte.

A manera de síntesis, se presenta una tabla donde se observan las solicitudes que se han recibido en el PACE de manera formal y que han permitido la participación de la instancia para emitir un criterio curricular. 
La asesoría del Programa de Apoyo Curricular y Evaluación de los Aprendizajes (PACE) en torno a la evaluación de los aprendizajes: alcances y retos desde la planificación curricular institucional

Ana Cristina Umaña, Xinia Calvo, Natalia Salas, Laura Jiménez, Suzanne Soto, Gisselle Gómez, Carolina Tencio y

Melba Fallas

Tabla 1. Apelaciones recibidas en el PACE en el periodo 2009-2013

\begin{tabular}{|l|l|l|}
\hline Solicitante & \multicolumn{1}{|c|}{ Tipo de apelación } & \multicolumn{1}{|c|}{$\begin{array}{c}\text { Fecha de } \\
\text { recepción de la } \\
\text { solicitud }\end{array}$} \\
\hline DEFE & $\begin{array}{l}\text { Diseño de pruebas escritas: Se expusieron casos en los que hubo } \\
\text { errores en la asignación de puntajes de los ítems de evaluación y } \\
\text { errores en la construcción de ítems. }\end{array}$ & Febrero 2009 \\
\hline DEFE & $\begin{array}{l}\text { De contenido: La prueba escrita evaluó una temática que no estaba } \\
\text { contemplada en las orientaciones académicas. }\end{array}$ & Marzo 2009 \\
\hline $\begin{array}{l}\text { Vicerrectoría } \\
\text { Académica }\end{array}$ & $\begin{array}{l}\text { Porcentaje de las actividades de evaluación: Se presentó una única } \\
\text { prueba escrita con un valor del 60\% que abarcó el contenido de la } \\
\text { asignatura y una tarea del 40\% de alta complejidad. }\end{array}$ & Marzo 2009 \\
\hline DEFE & $\begin{array}{l}\text { Validez de los instrumentos de evaluación: Tanto la prueba escrita } \\
\text { como la tarea de la asignatura no concordaron con el contenido de la } \\
\text { unidad didáctica. }\end{array}$ & Noviembre \\
\hline DEFE & $\begin{array}{l}\text { Diseño de la prueba escrita: El tiempo, la extensión y complejidad de } \\
\text { la prueba escrita no se relacionó con el tipo de temática evaluada. }\end{array}$ & Enero 2010 \\
\hline DEFE & $\begin{array}{l}\text { Diseño de la prueba escrita: El tiempo de aplicación no correspondió } \\
\text { con el requerido por el estudiantado. }\end{array}$ & Marzo 2011 \\
\hline Docente & $\begin{array}{l}\text { Criterios de evaluación: La forma en la que se le comunicaron al } \\
\text { estudiantado los criterios de evaluación y las instrucciones para las } \\
\text { actividades evaluativas generó confusión a la hora de realizarlas. }\end{array}$ & Julio 2013 \\
\hline DEFE & $\begin{array}{l}\text { Diseño de la prueba escrita: Se presentaron errores en la } \\
\text { construcción de ítems, en la elaboración de instrucciones y en los } \\
\text { criterios de evaluación. }\end{array}$ & Agosto 2013 \\
\hline Estudiante & $\begin{array}{l}\text { Diseño de pruebas escritas: se presentaron errores en la } \\
\text { construcción de ítems, así como en la relación entre lo que se } \\
\text { deseaba evaluar y el tipo de instrumento utilizado. }\end{array}$ & Febrero 2014 \\
\hline
\end{tabular}

Fuente: elaboración propia, 2014

A partir de las experiencias en la resolución de apelaciones el PACE ha logrado identificar las principales oportunidades de mejora y las áreas que en materia de evaluación de los aprendizajes requieren prioridad de atención a nivel institucional. Una de las acciones que el PACE ha llevado a cabo en busca de esa mejora que se menciona es la participación en diferentes procesos institucionales, los cuales se detallan a continuación. 
La asesoría del Programa de Apoyo Curricular y Evaluación de los Aprendizajes (PACE) en torno a la evaluación de los aprendizajes: alcances y retos desde la planificación curricular institucional

Ana Cristina Umaña, Xinia Calvo, Natalia Salas, Laura Jiménez, Suzanne Soto, Gisselle Gómez, Carolina Tencio y Melba Fallas

\section{Participación del PACE en procesos de mejora en el área de evaluación de los aprendizajes.}

Como parte de su gestión el PACE ha sido partícipe de procesos de construcción en tres ámbitos: producción académica, investigación y normativa institucional, logrando con ello procesos y productos que han fortalecido y orientado el quehacer académico de la UNED.

Estas acciones no solo han permitido un lenguaje común institucional, sino también el establecimiento de líneas de acción en un sistema educativo en el que, por su naturaleza, intervienen diversos agentes y momentos en el proceso de mediación docente, que demandan por tanto claridad en los fundamentos teóricoprácticos del currículo, en este caso en particular de la evaluación de los aprendizajes.

A continuación se esboza la participación que ha tenido el PACE en el desarrollo de procesos y productos que en esta materia se han obtenido.

\section{Producción académica.}

EI PACE, junto a otras instancias académicas, ha logrado concretar una serie de documentos cuyo propósito es orientar las acciones en el marco de la evaluación de los aprendizajes en la UNED. Lo que ha permitido profundizar en la temática y brindarle a la academia las herramientas para su gestión en este ámbito.

La construcción de estos documentos se ha llevado a cabo bajo una línea de trabajo de discusión de propuestas que involucra las experiencias en el campo de la mediación docente desde el conocimiento de académicos y especialistas, 
La asesoría del Programa de Apoyo Curricular y Evaluación de los Aprendizajes (PACE) en torno a la evaluación de los aprendizajes: alcances y retos desde la planificación curricular institucional

Ana Cristina Umaña, Xinia Calvo, Natalia Salas, Laura Jiménez, Suzanne Soto, Gisselle Gómez, Carolina Tencio y Melba Fallas

característica que permea a cada uno de ellos. Lo anterior garantiza que estos sean pertinentes y funcionales para su uso en los diferentes espacios universitarios que así lo requieran.

Los equipos que participaron en conjunto con el PACE en la construcción de los documentos fueron el Programa de Aprendizaje en Línea (PAL) y la Comisión Institucional de Evaluación de los Aprendizajes (CIEA), coordinada por el PACE y conformada por las personas representantes de cada una de las escuelas de la universidad y del Sistema de Estudios de Posgrado.

La producción académica contempla tanto orientaciones que atienden los fundamentos teóricos correspondientes como su aplicación. Así, por ejemplo, se elaboraron documentos sobre instrumentos de evaluación (pruebas escritas, lista de cotejo, matrices de evaluación) y la guía de procedimientos específicos llamada La aplicación de instrumentos de evaluación presencial para poblaciones estudiantiles específicas, en la que participó un equipo conformado por la CIEA, la Vicerrectoría Académica, el Centro de Operaciones Académicas y el PACE, entre otros documentos que tratan la temática desde una perspectiva teórica y que buscan explicar los fundamentos que la UNED aplica desde su normativa.

Se considera oportuno presentar la siguiente síntesis de las producciones que se han mencionado anteriormente y otras que por razones de espacio no se han podido destacar, sin embargo, se consideran documentos que han contribuido y seguirán contribuyendo en la gestión académica sobre el tema de la evaluación de los aprendizajes. 
La asesoría del Programa de Apoyo Curricular y Evaluación de los Aprendizajes (PACE) en torno a la evaluación de los aprendizajes: alcances y retos desde la planificación curricular institucional

Ana Cristina Umaña, Xinia Calvo, Natalia Salas, Laura Jiménez, Suzanne Soto, Gisselle Gómez, Carolina Tencio y

Melba Fallas

Tabla 2. Producciones académicas del PACE en materia de evaluación de los aprendizajes (2009-2013)*

\begin{tabular}{|c|c|}
\hline Año & Producciones \\
\hline 2007-2008 & 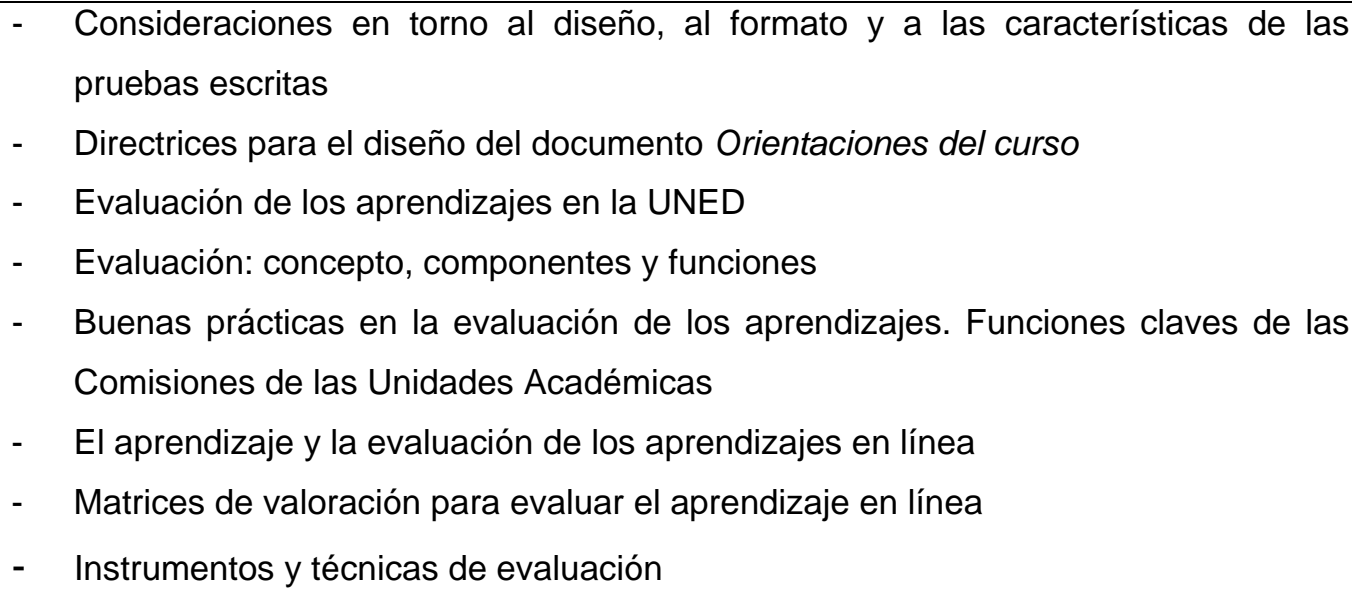 \\
\hline 2009-2010 & $\begin{array}{l}\text { - Documento de consideraciones sobre los aspectos contenidos en la normativa } \\
\text { institucional en relación con el tema de la evaluación de los aprendizajes } \\
\text { - } \quad \text { Informe sobre los artículos del Reglamento General Estudiantil (UNED, 2012) en } \\
\text { relación con la evaluación de los aprendizajes (Solicitud del Consejo Universitario } \\
\text { REF-CU-165-2009) } \\
\text { - } \quad \text { Funciones claves de la Comisión Institucional de Evaluación } \\
\text { - } \quad \text { Lineamientos generales en materia de evaluación de los aprendizajes } \\
\text { - } \quad \text { Investigación sobre pruebas escritas aplicadas en el periodo comprendido entre } \\
\text { - } 2005-2007 \\
\text { - } \quad \text { Analuálisis de los informes de equipo base del periodo } 2009 \\
\text { - Consideraciones en relación con el uso de estrategias, técnicas y herramientas } \\
\text { - Intilizadas en la evaluación auténtica } \\
\text { - Instrumentos de registro. Matrices de evaluación } \\
\text { Documento de las funciones claves de la Comisión Institucional de los } \\
\text { Aprendizajes }\end{array}$ \\
\hline & $\begin{array}{l}\text { - Procedimiento para la gestión de la aplicación de instrumentos de evaluación } \\
\text { presencial a poblaciones estudiantiles específicas } \\
\text { - Procedimiento para la gestión operativa de la aplicación de instrumentos de } \\
\text { evaluación de los aprendizajes presenciales por situaciones de fuerza mayor }\end{array}$ \\
\hline
\end{tabular}


La asesoría del Programa de Apoyo Curricular y Evaluación de los Aprendizajes (PACE) en torno a la evaluación de los aprendizajes: alcances y retos desde la planificación curricular institucional

Ana Cristina Umaña, Xinia Calvo, Natalia Salas, Laura Jiménez, Suzanne Soto, Gisselle Gómez, Carolina Tencio y

Melba Fallas

\begin{tabular}{|c|c|}
\hline 2011-2013 & $\begin{array}{l}\text { - Procedimiento para la gestión operativa de los instrumentos de evaluación de los } \\
\text { aprendizajes } \\
\text { - Modificación del Reglamento General Estudiantil (UNED, 2012): evaluación de los } \\
\text { aprendizajes } \\
\text { - } \quad \text { Análisis de los informes de apelaciones SAI 2010-2011 } \\
\text { - El carácter riguroso de la evaluación de los aprendizajes: delimitaciones } \\
\text { conceptual y operativa de los criterios de calidad que la determinan. } \\
\text { - Criterios para la elaboración de la lista de cotejo, escalas de calificación y matrices } \\
\text { de valoración }\end{array}$ \\
\hline
\end{tabular}

Fuente: Elaboración propia de las autoras

Nota: *En conjunto con otras instancias institucionales

Es importante destacar que para elaborar los diferentes documentos se ha recurrido a la investigación y al estudio profundo de la temática, con el fin de fundamentar las propuestas que se han generado desde el Programa o bien en conjunto con otras instancias académicas.

Cabe mencionar que entre los años 2007-2009 se realizó un proyecto de investigación, en coordinación con los miembros de la CIEA, sobre pruebas escritas aplicadas en el periodo comprendido entre los años 2005-2007 en ocho asignaturas que se identificaron como problemáticas por los indicadores de reprobación y el número de apelaciones recibidas. Se presentó así un informe solicitado por la Vicerrectoría Académica para tal fin.

Es así como el PACE, desde su función de asesoría en el área curricular, ha logrado articular, generar y coadyuvar en esta materia, en la ejecución de acciones en pro de la calidad de los procesos de aprendizaje de la UNED. Además, ha promovido espacios para la presentación de experiencias sobre las temáticas que se han compartido con la comunidad universitaria. Sobre este particular, en el año 2008 el PACE propuso y llevó a cabo el taller Compartiendo 
La asesoría del Programa de Apoyo Curricular y Evaluación de los Aprendizajes (PACE) en torno a la evaluación de los aprendizajes: alcances y retos desde la planificación curricular institucional

Ana Cristina Umaña, Xinia Calvo, Natalia Salas, Laura Jiménez, Suzanne Soto, Gisselle Gómez, Carolina Tencio y Melba Fallas

Experiencias y Gestando Cambios. La Evaluación de los Aprendizajes en Entornos Virtuales, en el cual se propuso que las personas docentes y encargadas de las cátedras de las cuatro escuelas presentaran sus buenas prácticas desde la evaluación de los aprendizajes desde la virtualidad.

Aunado a lo anterior, se ha coordinado con el Centro de Capacitación en Educación a Distancia (CECED) para llevar a cabo capacitaciones y talleres, espacios donde se utilizarán y divulgarán estos documentos.

\section{Participación en procesos de mejora en la normativa institucional.}

EI PACE, como instancia asesora en la evaluación de los aprendizajes, también ha participado en diferentes espacios en los que esta ha sido tema de discusión, llevando a cabo procesos de análisis de la normativa institucional en pro de garantizar los derechos y deberes del estudiantado.

Sobre este particular, en el año 2009 se inició el planteamiento de mejoras del Reglamento General Estudiantil (UNED, 2012), en el que se presentó el criterio, desde la CIEA, solicitado por el Consejo Universitario, puntualmente en:

- El Informe sobre los artículos del Reglamento General Estudiantil en relación con la evaluación de los aprendizajes (Solicitud del Consejo Universitario REF-CU-165-2009)

- Los lineamientos generales en materia de evaluación de los aprendizajes

En cuanto a este último punto los aspectos tratados fueron los siguientes:

- Consideraciones en relación con la evaluación de los aprendizajes contemplados en el Reglamento General Estudiantil

- Aspectos por considerar en la evaluación de los aprendizajes en la UNED 
La asesoría del Programa de Apoyo Curricular y Evaluación de los Aprendizajes (PACE) en torno a la evaluación de los aprendizajes: alcances y retos desde la planificación curricular institucional

Ana Cristina Umaña, Xinia Calvo, Natalia Salas, Laura Jiménez, Suzanne Soto, Gisselle Gómez, Carolina Tencio y

Melba Fallas

- Figuras e instancias involucradas en los procesos de evaluación de los aprendizajes

- Integración del capítulo de trabajos finales de graduación (TFG) al Reglamento General Estudiantil

Asimismo, en los años 2012 y 2013 se le realizaron modificaciones al Reglamento General Estudiantil en su "Capítulo VIII. Evaluación de los aprendizajes", donde los aspectos tratados fueron los trabajos finales de graduación y la evaluación de los aprendizajes, respectivamente. En el año 2013 se conformó un equipo junto a la Comisión de Jurídicos del Consejo Universitario, integrada por representantes de la Oficina de Registro, la Vicerrectoría Académica, la Dirección de Extensión Universitaria y el PACE donde se da un replanteamiento de dicho capítulo.

Estos y otros procesos de discusión y propuestas son parte de las acciones de articulación y generación de cambios de los que el PACE ha sido partícipe, en procura del fortalecimiento de la evaluación de los aprendizajes.

\section{Retos del PACE en materia de evaluación de los aprendizajes}

El desarrollo del tema de evaluación de los aprendizajes, así como las posibles contribuciones que desde la instancia se pueden hacer para el posicionamiento y puesta en práctica de los principios orientadores en dicha materia, siempre ha estado enmarcado por dos aspectos que a la fecha siguen siendo medulares: a) el contexto institucional y la normativa y b) las condiciones propias de desarrollo del programa. Ambos factores son fundamentales en el análisis de la ruta a seguir en el PACE y, sobre todo, en los retos que la dependencia afronta en el marco de las intenciones propias de la universidad. 
La asesoría del Programa de Apoyo Curricular y Evaluación de los Aprendizajes (PACE) en torno a la evaluación de los aprendizajes: alcances y retos desde la planificación curricular institucional

Ana Cristina Umaña, Xinia Calvo, Natalia Salas, Laura Jiménez, Suzanne Soto, Gisselle Gómez, Carolina Tencio y Melba Fallas

En relación con el contexto institucional, el Plan Académico 2012-2017 (2013) marca la ruta a seguir en los próximos cinco años para la gestión académica institucional. En dicho documento se propone que la evaluación de los aprendizajes debe trascender la mera medición para ser una oportunidad para aprender; además, esta es incorporada como una de las dimensiones del Plan, como línea estratégica y eje articulador en busca del "mejoramiento del quehacer sustantivo de la universidad" (p. 23) y en pro de la pertinencia y la calidad académicas. Además, el Reglamento para la Gestión Académica (2012) hace referencia al tema específicamente en los artículos 31 y 32, en los que se le asigna al PACE la atención, la asesoría y el acompañamiento en materia de evaluación de los aprendizajes.

Por otra parte, el proceso de consolidación de la instancia que ya cuenta con más de siete años de existencia, a través de los cuales ha logrado madurez y experiencia en la asesoría y la formación profesional de sus colaboradores, le permite en la actualidad hacer frente a los retos y las acciones que en torno al tema de la evaluación de los aprendizajes se plantea la UNED en el Plan Académico 2012-2017, entre los cuales se destacan:

$>$ Una evaluación auténtica y transparente

$>$ El desarrollo de procesos de evaluación integral en todo el proceso de aprendizaje

> Evaluar como sinónimo de regulación de los aprendizajes

> Instrumentos que se caractericen por su validez, porque están técnicamente elaborados y correctamente aplicados en relación con las realizaciones del estudiante.

> Consolidar la evaluación de tipo formativo, entendida como la acción reguladora y autorreguladora de los aprendizajes 
La asesoría del Programa de Apoyo Curricular y Evaluación de los Aprendizajes (PACE) en torno a la evaluación de los aprendizajes: alcances y retos desde la planificación curricular institucional

Ana Cristina Umaña, Xinia Calvo, Natalia Salas, Laura Jiménez, Suzanne Soto, Gisselle Gómez, Carolina Tencio y Melba Fallas

> Propiciar la coevaluación entre pares para cumplir los principios de construcción conjunta y colaboradora de los aprendizajes, de manera que el estudiantado cuente con la oportunidad de contrastar sus conceptos y modelos mentales con los de sus pares, como miembros de una verdadera comunidad de aprendizaje.

> Trascender los métodos tradicionales que han favorecido la memorización de contenidos para dar paso a la elaboración de los materiales educativos y el uso de recursos y medios didácticos alternativos.

Ante los retos y las acciones antes mencionados, y dado que a la fecha la oferta curricular de la universidad se encuentra mayoritariamente actualizada, el panorama actual le permite al PACE atender el tema de la evaluación de los aprendizajes desde la perspectiva de la evaluación auténtica con mayor propiedad, esto debido a que las condiciones del contexto y el proceso de desarrollo de la instancia se convierten en elementos medulares para tal fin.

\section{Conclusiones}

A partir de la experiencia de estos siete años se puede concluir lo siguiente:

- La asesoría curricular en diseño de planes de estudios y asignaturas en materia de evaluación de los aprendizajes debe establecer un vínculo entre las necesidades propias del campo específico que se trate y la pertinencia curricular por la naturaleza de la educación a distancia.

- EI PACE constituye dentro de la universidad una instancia que puede articular conexiones con otras dependencias para optimizar el quehacer académico en materia de apelaciones, velando por la calidad de la educación que se le ofrece al estudiantado. Así como atender de manera directa a personas interesadas 
La asesoría del Programa de Apoyo Curricular y Evaluación de los Aprendizajes (PACE) en torno a la evaluación de los aprendizajes: alcances y retos desde la planificación curricular institucional

Ana Cristina Umaña, Xinia Calvo, Natalia Salas, Laura Jiménez, Suzanne Soto, Gisselle Gómez, Carolina Tencio y Melba Fallas

de la comunidad universitaria en mejorar procesos curriculares y de evaluación de los aprendizajes.

- En la experiencia de la asesoría curricular del PACE se evidencia la necesidad de que las personas responsables de los procesos educativos de la universidad cuenten con mayor formación sobre las teorías de evaluación, así como sobre la normativa institucional vigente sobre este tema.

- La producción académica en materia de evaluación de los aprendizajes que se ha logrado con la participación del PACE y otras instancias de la universidad ha contribuido a brindar solidez y pertinencia al proceso de evaluación de los aprendizajes en la formación profesional que se lleva a cabo desde un sistema a distancia.

- El PACE, como instancia asesora en la temática de evaluación de los aprendizajes, se enfrenta constantemente a retos desde las tendencias institucionales, nacionales e internacionales que demandan formación continua.

\section{Recomendaciones}

Como recomendaciones se proponen las siguientes:

- El PACE, como instancia asesora en materia de evaluación de los aprendizajes, debe continuar generando espacios académicos con intención de divulgar las producciones académicas que se realizan sobre la temática y favorecer la formación de la comunidad universitaria.

- Es necesario que el PACE continúe generando articulaciones con las diferentes unidades académicas para atender las demandas y los retos de la universidad.

- El PACE debe continuar desarrollando proyectos de investigación, capacitándose y gestionando pasantías e intercambios con homólogos o 
La asesoría del Programa de Apoyo Curricular y Evaluación de los Aprendizajes (PACE) en torno a la evaluación de los aprendizajes: alcances y retos desde la planificación curricular institucional

Ana Cristina Umaña, Xinia Calvo, Natalia Salas, Laura Jiménez, Suzanne Soto, Gisselle Gómez, Carolina Tencio y Melba Fallas

especialistas en la temática, tanto a nivel nacional como internacional, para mantenerse actualizado sobre el tema de la evaluación de los aprendizajes.

\section{Referencias bibliográficas}

Calvo, X., Fallas, M., Gómez, G., Jiménez, L., Salas, N., Robles, L., Soto, S., Tencio, C. y Umaña, A. C. (2011). Asesoría Curricular del diseño de planes de estudios y de cursos en la UNED de Costa Rica: Una experiencia de 3 años. Ponencia presentada en el XV Congreso Internacional de Tecnología y Educación a Distancia. Desafíos e innovaciones de la educación superior frente al estudiante del Siglo XXI. UNED, Costa Rica.

Camelo, G. (2012). Evaluación curricular de la carrera de Farmacia en la Facultad de Ciencias de la Universidad Nacional de Colombia, a partir de la perspectiva del egresado. Instituto de Investigación en Educación, Facultad de Ciencias Humanas, Universidad Nacional de Colombia. Bogotá, Colombia.

Programa de Apoyo Curricular y Evaluación de los Aprendizajes (2013). Glosario de términos curriculares para la Universidad Estatal a Distancia. UNED, San José, Costa Rica.

Universidad Estatal a Distancia (2004). Modelo Pedagógico de la Universidad Estatal a Distancia. San José, Costa Rica: EUNED.

Universidad Estatal a Distancia (2005). Reglamento para la Gestión Académica de la UNED. UNED, San José, Costa Rica.

Universidad Estatal a Distancia (2007). Plan Estratégico del Programa de Apoyo Curricular y Evaluación de los Aprendizajes 2007-2012 (material mimeografiado). UNED, San José, Costa Rica.

Universidad Estatal a Distancia (2012). Reglamento General Estudiantil. UNED, San José, Costa Rica.

Universidad Estatal a Distancia (2013) Plan de Desarrollo Académico 2012-2017. UNED, San José, Costa Rica. 